\title{
A Correlation and Path Analysis Studies of Quantitative and Qualitative Traits in Tomato
}

\author{
Chandan Kumar* and Dheeraj Singh
}

ICAR-Central Arid Zone Research Institute, KVK, Pali-Marwar-306 401 (Rajasthan), India

*Corresponding author

\section{A B S T R A C T}

\section{Keywords}

Correlation, Path analysis, Tomato

\section{Article Info}

Accepted:

20 March 2018

Available Online:

10 April 2018
Correlation and path analysis were carried out in twenty eight tomato genotypes for quantitative and qualitative characters. The association studies showed that fruit yield per plant was genotypic and phenotypic positive correlated with number of flowers per plant, number of fruits per cluster, fruit diameter, fruit length, average fruit weight, number of locules per fruit and seed yield per plant. Path analysis studies done to study the cause and effect relationship revealed number of seeds per fruit, number of clusters per plant, fruit diameter and number of fruits per plant exerted positive direct effects on fruit yield per plant at genotypic level. However, phenotypic level, maximum exerted positive direct effects on fruit yield per plant was number of seeds per fruit. So, by improving these traits yield can be significantly increased.

\section{Introduction}

Tomato (Lycopersicum esculentum L.) is one of the most important vegetable crops grown throughout the world because of its wider adaptability, high yielding potential and suitability for variety of uses in fresh as well as processed food industries (Kumar and Singh, 2016a).

Exploring natural diversity as a source of novel alleles to improve the productivity, quality and nutritional value of the crop is the base line of any breeding programme (Kumar and Singh, 2016b). Yield is a complex character and selection for yield and yield components deserves considerable attention. A crop breeding programme, aimed at increasing the plant productivity requires consideration not only of yield but also of its components that have direct or indirect bearing on yield. Information on association of characters, direct and indirect effects contributed by each character towards yield will be an added advantage in aiding the selection process (Kumar and Singh, 2017c).

Correlation and path analysis establish the extent of association between yield and its components and also bring out relative importance of their direct and indirect effects, thus giving an obvious understanding of their association with fruit yield. Ultimately, this kind of analysis could help the breeder to design his selection strategies to improve fruit yield and nutritional value (Alirwar et al., 2013). In the light of the above scenario, the present investigation was carried out with the 
objective of studying the character associations in tomato for quantitative and qualitative value.

\section{Materials and Methods}

A field experiment was conducted during rabi season of 2013-14 on sandy loam soil at Vegetable Research Farm, BHU, Varanasi, India which is situated at $83.03^{\circ} \mathrm{E}$ longitude and $25.02^{\circ} \mathrm{N}$ latitude at an altitude of 128.93 $\mathrm{m}$ above mean sea level in the North gangetic zone. The Eight genetically diverse lines [Arka Meghali, Pant T-3, Punjab Chhuhara, H-88-78-1, Arka Alok, Azad T-5, H-24 (Hisar Anmol), Sel-7 (Hisar Arun)] was procured from IIVR, Varanasi and crossed in diallel mating design during rabi 2012-13. The resultant $28 \quad \mathrm{~F}_{1}$ 's were evaluated along with their parents was during rabi 2013-14 in randomized block design. In each replication, randomly 5 plants in each genotype were marked for observation. Observations were recorded for nineteen qualitative and quantitative characters viz., days to first flowering, number of flowers per cluster, days to first harvest, number of clusters per plant, number of fruit per cluster, fruit diameter $(\mathrm{cm})$, fruit length $(\mathrm{cm})$, average fruit weight $(\mathrm{g})$, number of fruits per plant, number of locules per fruit, plant height at final harvest $(\mathrm{cm})$, pericarp thickness $(\mathrm{cm})$, number of branches per plant, number of seed per fruit, yield per plant (kg.), total soluble solids ( ${ }^{\circ}$ Brix), ascorbic acid (mg/100g), acidity as anhydrous citric acid (\%), lycopene content (mg/100g).

For estimation of quality traits, ripe fruits were selected randomly. Total soluble solids was estimated by using hand refractometer, ascorbic acid and lycopene content was estimated according to procedure given by Ranganna (1986), titrable acidity will be measure based on the titration of tomato acid mainly citric acid, by an alkaline solution. The phenotypic and genotypic coefficients of correlation were computed by following AlJibouri et al., (1958). The path coefficient analysis was carried out using phenotypic correlation values of yield components on yield as suggested by Wright (1921) and illustrated by Dewey and Lu (1959).

\section{Results and Discussion}

\section{Correlation coefficient analysis}

In general it was found that genotypic correlation coefficients were higher in magnitude than their corresponding phenotypic values indicating thereby a strong inherent association between various traits under study (Alirwar et al., 2013). Basically yield is the main character with which all other characters are positively or negatively correlated. Days to first flowering which had been found positive significant genotypically and phenotypically correlated with days to first harvest, average fruit weight and pericarp thickness, whereas negatively significant with other traits. Number of flowers per cluster exhibited positive correlation with number of clusters per plant, number of fruit per cluster, fruit length, number of seeds per fruit, yield per plant and TSS while it showed a negative genotypic significant association with days to first harvest and lycopene content.

Days to first harvest exhibited positive significant (genotypic) correlation with pericarp thickness whereas it showed a negative significant association with other most characters. Number of clusters per plant showed positive significant with number of fruits per cluster, fruit diameter, fruit length, number of fruits per plant, number of locules per fruit, number of branches per plant, number of seeds per fruit, yield per plant and TSS content, it showed negative and significant association with average fruit weight and pericarp thickness at genotypic level. 
Number of fruits per cluster had been positive correlated with fruit length, average fruit weigh, plant height, pericarp thickness, number of seeds per fruit, yield per plant, TSS and lycopene content. Fruit diameter showed positive significant correlation with number of fruits per plant, number of locules per fruit, plant height, number of seeds per fruit, yield per plant, ascorbic acid, acidity and lycopene content. Fruit length exhibited positive significant genotypic and phenotypic correlation with average fruit weight, number of branches per plant, number of seeds per fruit, yield per plant and TSS, whereas negative significant genotypic and phenotypic correlation were discovered with ascorbic acid and lycopene content.

Average fruit weight exhibited positive significant genotypic and phenotypic correlation with pericarp thickness, number of seeds per fruit, yield per plant and TSS whereas, negative significant genotypic and phenotypic correlation were examined with number of fruits per plant, number of locules per fruit, number of branches per plant, ascorbic acid and lycopene content. Number of fruits per plant showed positive significant genotypic and phenotypic correlation with number locules per plant, number of branches per plant, ascorbic acid and lycopene content, while negative significant genotypic and phenotypic correlation were seen only with pericarp thickness.

Number of locules per fruit showed positive significant genotypic and phenotypic correlation with number of branches per plant, number of seeds per fruit, yield per plant, ascorbic acid and lycopene content, whereas negative significant genotypic and phenotypic correlation were observed with pericarp thickness and TSS. Plant height exhibited positive significant genotypic and phenotypic correlation with pericarp thickness, TSS and lycopene content. Pericarp thickness showed positive significant genotypic and phenotypic correlation only with TSS. The negative significant genotypic and phenotypic correlation was discovered with number of branches per plant, number of seeds per fruit, yield per plant, ascorbic acid and acidity.

Number of branches per plant exhibited positive significant genotypic and phenotypic correlation with number of seeds per fruit and acidity. Number of seed per plant showed positively significant association with yield per plant and TSS, while no negative significant genotypic and phenotypic correlation exited with any parameters.

The yield per plant showed only genotypic positively significant association with TSS and non-significant negative association with any characters. TSS exhibited negative significant association with ascorbic acid and acidity. Ascorbic acid exhibited positive significant association with acidity and lycopene content. Acidity and lycopene were was found to show non-significant association with any character.

The results are in accordance with Reddy et al., (2013) for fruit width with fruit yield per plant, number of primary branches per plant and number of flowers per cluster had significantly positive correlation with plant height and days to last fruit harvest and shelf life had significant negative relationship with fruit yield per plant. Similar results are also observed by Alirwar et al., (2013), number of fruits per cluster and ascorbic acid had significantly positive association with number of primary branches per plant and days to $50 \%$ flowering while, days to last fruit harvest had negative association with number of primary branches per plant. Results are in also accordance with Laxmi et al., (2017). Days to first fruit set, days to first fruit harvest and shelf life had positive association with days to $50 \%$ flowering. 
Table.1 Genotypic correlation coefficient between different quantitative and qualitative characters of tomato

\begin{tabular}{|c|c|c|c|c|c|c|c|c|c|c|c|c|c|c|c|c|c|c|}
\hline Parameters & $\begin{array}{c}\text { No. of } \\
\text { flowers/ } \\
\text { cluster }\end{array}$ & $\begin{array}{c}\text { Days } \\
\text { to1 } \\
\text { harvest }^{\text {st }}\end{array}$ & $\begin{array}{c}\text { No. of } \\
\text { clusters/ } \\
\text { plant }\end{array}$ & $\begin{array}{l}\text { No. of } \\
\text { fruit/ } \\
\text { cluster }\end{array}$ & $\begin{array}{l}\text { Fruit } \\
\text { dia. } \\
(\mathrm{cm})\end{array}$ & $\begin{array}{c}\text { Fruit } \\
\text { length } \\
(\mathrm{cm})\end{array}$ & $\begin{array}{c}\text { Avg } \\
\text { fruit } \\
\text { wt. (g.) }\end{array}$ & $\begin{array}{l}\text { No. of } \\
\text { fruits/ } \\
\text { plant }\end{array}$ & $\begin{array}{c}\text { No. of } \\
\text { locules/ } \\
\text { fruit }\end{array}$ & $\begin{array}{l}\text { Plant } \\
\text { ht. } \\
(\mathrm{cm})\end{array}$ & $\begin{array}{c}\text { Pericarp } \\
\text { thickness } \\
(\mathrm{cm})\end{array}$ & $\begin{array}{c}\text { No. of } \\
\text { branches/ } \\
\text { plant }\end{array}$ & $\begin{array}{l}\text { No. of } \\
\text { seeds/ } \\
\text { fruit }\end{array}$ & $\begin{array}{l}\text { Yield/ } \\
\text { plant } \\
\text { (kg.) }\end{array}$ & $\begin{array}{c}\text { TSS } \\
\left({ }^{0} \text { Brix }\right)\end{array}$ & $\begin{array}{c}\text { Ascorbic } \\
\text { acid } \\
\text { (mg/100g } \\
\text { Fwt.) }\end{array}$ & $\begin{array}{l}\text { Acidity } \\
(\%)\end{array}$ & $\begin{array}{c}\text { Lycopene } \\
\text { (mg/100g } \\
\text { Fwt.) }\end{array}$ \\
\hline $\begin{array}{l}\text { Days to } 1^{\text {st }} \\
\text { flowering }\end{array}$ & $-0.249 * *$ & $1.001 * *$ & $-0.325 * *$ & $-0.511 * *$ & $-0.635^{* *}$ & -0.132 & $0.321 * *$ & $-1.141 * *$ & $-0.933 * *$ & 0.170 & $0.299 * *$ & 0.165 & -0.184 & $-0.629 * *$ & $-0.433 * *$ & $-0.636^{* *}$ & 0.096 & $-0.335 * \%$ \\
\hline $\begin{array}{l}\text { No. of } \\
\text { flowers/ } \\
\text { cluster }\end{array}$ & & $-0.242 * *$ & $0.504 * *$ & $0.510 * *$ & -0.124 & $0.349 * *$ & -0.104 & 0.121 & 0.143 & 0.178 & -0.145 & 0.157 & $0.214^{*}$ & $0.363^{* *}$ & $0.994 * *$ & -0.151 & -0.093 & $-0.222 \%$ \\
\hline $\begin{array}{l}\text { Days to } 1^{\mathrm{st}} \\
\text { harvest }\end{array}$ & & & $-0.554^{* *}$ & $-0.406^{* *}$ & $-0.517^{* *}$ & $-0.447^{* * *}$ & 0.180 & $-0.369^{* * *}$ & $-0.743 * *$ & $-0.297 * *$ & $0.234^{*}$ & $-0.625^{* *}$ & $-0.388^{* * *}$ & $-0.531^{\text {*** }}$ & $-0.291 * *$ & 0.029 & -0.043 & -0.039 \\
\hline $\begin{array}{l}\begin{array}{l}\text { No. of } \\
\text { clusters/ } \\
\text { plant }\end{array} \\
\end{array}$ & & & & $0.272 * *$ & $0.293 * *$ & $0.306^{* *}$ & $-0.258 * *$ & $0.418 * *$ & $0.475 * *$ & 0.037 & $-0.400 * *$ & $0.514 * *$ & $0.282 * *$ & $0.387 * *$ & $0.397 * *$ & -0.053 & 0.109 & -0.114 \\
\hline $\begin{array}{l}\text { No. of } \\
\text { fruit/cluster }\end{array}$ & & & & & -0.080 & $0.401 * *$ & $0.286^{* *}$ & 0.140 & 0.173 & $0.186^{*}$ & $0.205^{*}$ & 0.045 & $0.602 * *$ & $0.601 * *$ & $0.964 * *$ & -0.007 & -0.301 ** & $0.205^{*}$ \\
\hline $\begin{array}{l}\text { Fruit dia. } \\
(\mathrm{cm})\end{array}$ & & & & & & 0.030 & 0.126 & $0.192^{*}$ & $0.540 * *$ & $0.256 * *$ & -0.056 & -0.046 & $0.219^{*}$ & $0.256^{* *}$ & -0.333 ** & $0.230 *$ & $0.371 * *$ & $0.402^{* * *}$ \\
\hline $\begin{array}{l}\text { Fruit lgth. } \\
\text { (cm) }\end{array}$ & & & & & & & $0.417 * *$ & 0.057 & 0.099 & 0.100 & -0.008 & $0.330 * *$ & $0.360 * *$ & $0.487 * *$ & $0.570 * *$ & $-0.298 * *$ & -0.126 & $-0.605 * *$ \\
\hline $\begin{array}{l}\text { Avg fruit } \\
\text { wt. (g.) } \\
\end{array}$ & & & & & & & & $-0.508^{* *}$ & $-0.407 * *$ & 0.005 & $0.480^{* *}$ & $-0.369^{* * *}$ & $0.370^{* * *}$ & $0.248 * *$ & $0.202^{*}$ & $-0.422 * *$ & -0.098 & $-0.251 * *$ \\
\hline $\begin{array}{l}\text { No. of } \\
\text { fruits/ plant }\end{array}$ & & & & & & & & & $0.706^{* *}$ & -0.146 & $-0.415^{* * *}$ & $0.346^{* *}$ & -0.020 & 0.133 & -0.030 & $0.627^{* *}$ & 0.056 & $0.286 * *$ \\
\hline $\begin{array}{l}\text { No. of } \\
\text { locules/fruit }\end{array}$ & & & & & & & & & & 0.047 & $-0.480^{* * *}$ & $0.436 * *$ & $0.348^{* *}$ & $0.440^{* *}$ & $-0.277 * *$ & $0.465^{* *}$ & 0.118 & $0.601 * *$ \\
\hline $\begin{array}{l}\text { Plant ht. } \\
\text { (cm) }\end{array}$ & & & & & & & & & & & $0.197 *$ & -0.133 & -0.066 & 0.137 & $0.265^{* *}$ & -0.091 & 0.006 & $0.308 * *$ \\
\hline $\begin{array}{l}\text { Pericarp } \\
\text { thickness } \\
(\mathrm{cm})\end{array}$ & & & & & & & & & & & & -0.437 ** & $-0.234^{*}$ & $-0.248^{* *}$ & $0.778 * *$ & $-0.443^{* *}$ & -0.471 ** & 0.062 \\
\hline $\begin{array}{l}\text { No. of } \\
\text { branches/ } \\
\text { plant }\end{array}$ & & & & & & & & & & & & & $0.254 * *$ & 0.147 & -0.142 & 0.121 & $0.488^{* * *}$ & -0.000 \\
\hline $\begin{array}{l}\text { No. of } \\
\text { seeds/fruit }\end{array}$ & & & & & & & & & & & & & & $0.846^{* *}$ & $0.192 *$ & 0.096 & 0.085 & -0.077 \\
\hline $\begin{array}{l}\text { Yield/ plant } \\
\text { (Kg.) }\end{array}$ & & & & & & & & & & & & & & & $0.419 * *$ & 0.065 & -0.076 & -0.031 \\
\hline $\begin{array}{l}\text { TSS ( } \\
\text { Brix) }\end{array}$ & & & & & & & & & & & & & & & & $-0.634 * *$ & $-0.630^{* *}$ & -0.087 \\
\hline $\begin{array}{l}\text { Ascorbic } \\
\text { acid } \\
\text { (mg/100g } \\
\text { Fwt.) } \\
\end{array}$ & & & & & & & & & & & & & & & & & $0.449 * *$ & $0.602 * *$ \\
\hline $\begin{array}{l}\text { Acidity } \\
(\%)\end{array}$ & & & & & & & & & & & & & & & & & & 0.003 \\
\hline $\begin{array}{l}\text { Lycopene } \\
\text { (mg/100g } \\
\text { Fwt.) }\end{array}$ & & & & & & & & & & & & & & & & & & \\
\hline
\end{tabular}

$*$ Significant at $1 \%, * *$ Significant at 5\% 
Table.2 Phenotypic correlation coefficients between different quantitative and qualitative characters of tomato

\begin{tabular}{|c|c|c|c|c|c|c|c|c|c|c|c|c|c|c|c|c|c|c|}
\hline Parameters & $\begin{array}{l}\text { No. of } \\
\text { flowers/ } \\
\text { cluster }\end{array}$ & $\begin{array}{c}\text { Days } \\
\text { to } 1^{\text {st }} \\
\text { harvest }\end{array}$ & $\begin{array}{l}\text { No. of } \\
\text { clusters/ } \\
\text { plant }\end{array}$ & $\begin{array}{l}\text { No. of } \\
\text { fruit/ } \\
\text { cluster }\end{array}$ & $\begin{array}{c}\text { Fruit } \\
\text { dia. } \\
(\mathrm{cm})\end{array}$ & $\begin{array}{l}\text { Fruit } \\
\text { length } \\
(\mathrm{cm})\end{array}$ & $\begin{array}{c}\text { Avg } \\
\text { fruit } \\
\text { wt. (g.) }\end{array}$ & $\begin{array}{l}\text { No. of } \\
\text { fruits/ } \\
\text { plant }\end{array}$ & \begin{tabular}{|c|} 
No. of \\
locules/ \\
fruit
\end{tabular} & $\begin{array}{l}\text { Plant } \\
\text { ht. } \\
(\mathrm{cm})\end{array}$ & $\begin{array}{c}\text { Pericarp } \\
\text { thickness } \\
(\mathrm{cm})\end{array}$ & $\begin{array}{c}\text { No. of } \\
\text { branches/ } \\
\text { plant }\end{array}$ & $\begin{array}{l}\text { No. of } \\
\text { seeds/ } \\
\text { fruit }\end{array}$ & $\begin{array}{l}\text { Yield/ } \\
\text { plant } \\
\text { (kg.) }\end{array}$ & $\begin{array}{c}\text { TSS } \\
\left({ }^{0} \text { Brix }\right)\end{array}$ & $\begin{array}{c}\text { Ascorbic } \\
\text { acid } \\
\text { (mg/100g } \\
\text { Fwt.) }\end{array}$ & $\begin{array}{c}\text { Acidity } \\
(\%)\end{array}$ & $\begin{array}{c}\text { Lycopene } \\
\text { (mg/100g } \\
\text { Fwt.) }\end{array}$ \\
\hline $\begin{array}{l}\text { Days to } 1^{\text {st }} \\
\text { flowering }\end{array}$ & -0.081 & $0.222 *$ & -0.147 & -0.013 & -0.183 & 0.013 & 0.052 & $-0.355 * *$ & $-0.277 * *$ & 0.071 & 0.004 & -0.045 & -0.103 & $-0.212^{*}$ & -0.087 & -0.174 & 0.094 & -0.123 \\
\hline $\begin{array}{l}\text { No. of flowers/ } \\
\text { cluster }\end{array}$ & & -0.096 & $0.299 * *$ & $0.315^{\text {*** }}$ & -0.067 & 0.103 & -0.078 & 0.134 & 0.036 & 0.044 & -0.128 & 0.084 & 0.169 & $0.267 * *$ & 0.110 & -0.091 & -0.039 & -0.013 \\
\hline $\begin{array}{l}\text { Days to } 1^{\text {st }} \\
\text { harvest }\end{array}$ & & & $-0.299 * *$ & -0.167 & $-0.195 *$ & $-0.268 * *$ & 0.141 & $-0.214^{*}$ & $-0.355^{* *}$ & -0.181 & 0.111 & $-0.208^{*}$ & $-0.230^{*}$ & $-0.364 * *$ & $-0.226^{*}$ & 0.094 & 0.052 & -0.017 \\
\hline $\begin{array}{l}\text { No. of clusters/ } \\
\text { plant }\end{array}$ & & & & 0.133 & 0.097 & $0.187^{*}$ & -0.178 & $0.278 * *$ & $0.202 *$ & -0.037 & -0.177 & $0.267 * *$ & $0.217^{*}$ & $0.286^{* *}$ & 0.157 & -0.062 & -0.007 & -0.029 \\
\hline $\begin{array}{l}\text { No. of fruit/ } \\
\text { cluster }\end{array}$ & & & & & -0.010 & $0.245^{* *}$ & $0.200 *$ & 0.118 & 0.089 & 0.100 & 0.035 & 0.068 & $0.416^{* *}$ & $0.444 * *$ & $0.264 * *$ & 0.039 & $-0.202 *$ & 0.041 \\
\hline $\begin{array}{l}\text { Fruit dia. } \\
\text { (cm) }\end{array}$ & & & & & & 0.086 & 0.093 & 0.133 & $0.378 * *$ & 0.141 & -0.121 & 0.057 & $0.200^{*}$ & 0.180 & -0.164 & $0.286^{* *}$ & $0.244 * *$ & 0.175 \\
\hline $\begin{array}{l}\text { Fruit length } \\
(\mathrm{cm})\end{array}$ & & & & & & & $0.251 * *$ & -0.029 & 0.023 & 0.072 & -0.064 & 0.183 & $0.237^{*}$ & $0.245 * *$ & $0.334 * *$ & $-0.201 *$ & -0.065 & $-0.190 \%$ \\
\hline $\begin{array}{l}\text { Avg fruit wt. } \\
\text { (g.) }\end{array}$ & & & & & & & & $-0.459 * *$ & $-0.300 * *$ & -0.017 & $0.323 * *$ & $-0.232 *$ & $0.322 * *$ & $0.235^{*}$ & 0.073 & $-0.277 * *$ & -0.076 & -0.164 \\
\hline $\begin{array}{l}\text { No. of fruits/ } \\
\text { plant }\end{array}$ & & & & & & & & & $0.476^{* *}$ & -0.110 & $-0.341 * *$ & $0.231 *$ & -0.016 & 0.162 & -0.014 & $0.421 * *$ & 0.028 & 0.152 \\
\hline $\begin{array}{l}\text { No. of locules/ } \\
\text { fruit }\end{array}$ & & & & & & & & & & 0.062 & $-0.304 * *$ & $0.307^{* *}$ & $0.229 *$ & $0.303 * *$ & 0.015 & $0.319 * *$ & 0.003 & 0.095 \\
\hline $\begin{array}{l}\text { Plant ht. } \\
\text { (cm) }\end{array}$ & & & & & & & & & & & 0.111 & -0.052 & -0.045 & 0.100 & $0.197^{*}$ & -0.046 & 0.052 & 0.162 \\
\hline $\begin{array}{l}\text { Pericarp } \\
\text { thickness (cm) }\end{array}$ & & & & & & & & & & & & $-0.277 * *$ & -0.141 & -0.169 & $0.238^{*}$ & $-0.288 * *$ & $-0.227^{*}$ & -0.049 \\
\hline $\begin{array}{l}\text { No. of } \\
\text { branches/plant }\end{array}$ & & & & & & & & & & & & & $0.211^{*}$ & 0.119 & 0.038 & 0.170 & $0.266^{* *}$ & -0.143 \\
\hline $\begin{array}{l}\text { No. of seeds/ } \\
\text { fruit }\end{array}$ & & & & & & & & & & & & & & $0.766^{* *}$ & 0.080 & 0.083 & 0.086 & -0.030 \\
\hline $\begin{array}{l}\text { Yield/ plant } \\
\text { (Kg.) }\end{array}$ & & & & & & & & & & & & & & & 0.153 & 0.011 & -0.080 & -0.097 \\
\hline TSS $\left({ }^{0}\right.$ Brix $)$ & & & & & & & & & & & & & & & & $-0.244 * *$ & $-0.244 * *$ & 0.030 \\
\hline $\begin{array}{l}\text { Ascorbic acid } \\
\text { (mg/100g } \\
\text { Fwt.) } \\
\end{array}$ & & & & & & & & & & & & & & & & & $0.283^{* *}$ & $0.203 *$ \\
\hline $\begin{array}{l}\text { Acidity } \\
(\%)\end{array}$ & & & & & & & & & & & & & & & & & & 0.061 \\
\hline $\begin{array}{l}\text { Lycopene } \\
\text { (mg/100g } \\
\text { Fwt.) }\end{array}$ & & & & & & & & & & & & & & & & & & \\
\hline
\end{tabular}

* Significant at $1 \%, * *$ Significant at $5 \%$ 
Table.3 Direct (diagonal) and indirect effects of different qualitative and quantitative characters on yield in tomato at genotypic level

\begin{tabular}{|c|c|c|c|c|c|c|c|c|c|c|c|c|c|c|c|c|c|c|c|}
\hline Parameters & $\begin{array}{l}\text { Days to } \\
1^{\text {st }} \\
\text { flowering }\end{array}$ & $\begin{array}{l}\text { No. of } \\
\text { flowers/ } \\
\text { cluster }\end{array}$ & $\begin{array}{c}\text { Days } \\
\text { to1 }^{\text {st }} \\
\text { harvest }^{-1}\end{array}$ & $\begin{array}{l}\text { No. of } \\
\text { clusters/ } \\
\text { plant }\end{array}$ & $\begin{array}{l}\text { No. of } \\
\text { fruits/ } \\
\text { cluster }\end{array}$ & $\begin{array}{l}\text { Fruit } \\
\text { dia. } \\
(\mathrm{cm})\end{array}$ & $\begin{array}{c}\text { Fruit } \\
\text { length } \\
(\mathrm{cm})\end{array}$ & $\begin{array}{l}\text { Avg } \\
\text { fruit } \\
\text { wt. } \\
\text { (g.) }\end{array}$ & $\begin{array}{l}\text { No. of } \\
\text { fruits/ } \\
\text { plant }\end{array}$ & $\begin{array}{l}\text { No. of } \\
\text { locules/ } \\
\text { fruit }\end{array}$ & $\begin{array}{l}\text { Plant } \\
\text { ht. } \\
\text { (cm) }\end{array}$ & $\begin{array}{c}\text { Pericarp } \\
\text { thickness } \\
(\mathrm{cm})\end{array}$ & $\begin{array}{c}\text { No. of } \\
\text { branches/ } \\
\text { plant }\end{array}$ & $\begin{array}{c}\begin{array}{c}\text { No. } \\
\text { of } \\
\text { seeds/ } \\
\text { fruit }\end{array} \\
\end{array}$ & $\begin{array}{c}\text { TSS } \\
\left(\begin{array}{c}\text { To } \\
\text { Brix })\end{array}\right.\end{array}$ & $\begin{array}{c}\text { Ascorbic } \\
\text { acid } \\
\text { (mg/100g } \\
\text { Fwt.) }\end{array}$ & $\begin{array}{l}\text { Acidity } \\
(\%)\end{array}$ & $\begin{array}{c}\text { Lycopene } \\
\text { (mg/100g } \\
\text { Fwt.) }\end{array}$ & $\begin{array}{c}\text { R value } \\
\text { (genotypic } \\
\text { correlation) } \\
\text { with yield }\end{array}$ \\
\hline $\begin{array}{l}\text { Days to } 1^{\mathrm{st}} \\
\text { flowering }\end{array}$ & 0.117 & -0.030 & -0.007 & 0.050 & -0.226 & -0.183 & -0.009 & -0.073 & -0.229 & -0.117 & 0.021 & -0.018 & -0.027 & -0.114 & 0.062 & 0.074 & -0.009 & 0.088 & -0.629 \\
\hline $\begin{array}{l}\begin{array}{l}\text { No. of } \\
\text { flowers/ } \\
\text { cluster }\end{array} \\
\end{array}$ & -0.029 & 0.120 & 0.002 & -0.078 & 0.225 & -0.036 & 0.024 & 0.024 & 0.024 & 0.018 & 0.022 & 0.009 & -0.026 & 0.132 & -0.143 & 0.018 & 0.008 & 0.059 & 0.363 \\
\hline $\begin{array}{l}\text { Days to } 1^{\mathrm{st}} \\
\text { harvest }\end{array}$ & 0.117 & -0.029 & -0.007 & 0.085 & -0.179 & -0.149 & -0.031 & -0.041 & -0.074 & -0.093 & -0.036 & -0.014 & 0.102 & -0.240 & 0.042 & -0.003 & 0.004 & 0.010 & -0.531 \\
\hline $\begin{array}{l}\begin{array}{l}\text { No. of } \\
\text { clusters/ } \\
\text { plant }\end{array} \\
\end{array}$ & -0.038 & 0.061 & 0.004 & -0.154 & 0.120 & 0.084 & 0.021 & 0.058 & 0.084 & 0.059 & 0.004 & 0.024 & -0.084 & 0.174 & -0.057 & 0.006 & -0.010 & 0.030 & 0.387 \\
\hline $\begin{array}{l}\text { No. of } \\
\text { fruits/ } \\
\text { cluster }\end{array}$ & -0.060 & 0.061 & 0.003 & -0.042 & 0.442 & -0.023 & 0.027 & -0.065 & 0.028 & 0.022 & 0.023 & -0.012 & -0.007 & 0.372 & -0.139 & 0.001 & 0.027 & -0.054 & 0.601 \\
\hline $\begin{array}{l}\text { Fruit dia. } \\
(\mathrm{cm})\end{array}$ & -0.074 & -0.015 & 0.004 & -0.045 & -0.035 & 0.288 & 0.002 & -0.028 & 0.039 & 0.067 & 0.031 & 0.003 & 0.007 & 0.135 & 0.048 & -0.027 & -0.034 & -0.106 & 0.256 \\
\hline $\begin{array}{l}\text { Fruit } \\
\text { length(cm) } \\
\end{array}$ & -0.015 & 0.042 & 0.003 & -0.047 & 0.177 & 0.008 & 0.068 & -0.094 & 0.012 & 0.012 & 0.012 & 0.000 & -0.054 & 0.223 & -0.082 & 0.035 & 0.011 & 0.160 & 0.487 \\
\hline $\begin{array}{l}\text { Avg. fruit } \\
\text { wt. (g.) }\end{array}$ & 0.038 & -0.013 & -0.001 & 0.040 & 0.126 & 0.036 & 0.029 & -0.227 & -0.102 & -0.051 & 0.001 & -0.029 & 0.060 & 0.229 & -0.029 & 0.049 & 0.009 & 0.066 & 0.248 \\
\hline $\begin{array}{l}\text { No. of } \\
\text { fruits/plant }\end{array}$ & -0.134 & 0.015 & 0.003 & -0.064 & 0.062 & 0.055 & 0.004 & 0.115 & 0.201 & 0.088 & -0.018 & 0.025 & -0.057 & -0.012 & 0.004 & -0.073 & -0.005 & -0.076 & 0.133 \\
\hline $\begin{array}{l}\text { No. of } \\
\text { locules/ } \\
\text { fruit }\end{array}$ & -0.109 & 0.017 & 0.005 & -0.073 & 0.077 & 0.155 & 0.007 & 0.092 & 0.142 & 0.125 & 0.006 & 0.029 & -0.071 & 0.215 & 0.040 & -0.054 & -0.011 & -0.158 & 0.440 \\
\hline $\begin{array}{l}\text { Plant ht. } \\
(\mathrm{cm})\end{array}$ & 0.020 & 0.021 & 0.002 & -0.006 & 0.082 & 0.074 & 0.007 & -0.001 & -0.029 & 0.006 & 0.122 & -0.012 & 0.022 & -0.041 & -0.038 & 0.011 & -0.001 & -0.081 & 0.137 \\
\hline $\begin{array}{l}\text { Pericarp } \\
\text { thickness } \\
(\mathrm{cm})\end{array}$ & 0.035 & -0.017 & -0.002 & 0.062 & 0.091 & -0.016 & -0.001 & -0.109 & -0.083 & -0.060 & 0.024 & -0.061 & 0.072 & -0.144 & -0.112 & 0.051 & 0.043 & -0.016 & -0.248 \\
\hline $\begin{array}{l}\text { No. of } \\
\text { branches/ } \\
\text { plant }\end{array}$ & 0.019 & 0.019 & 0.005 & -0.079 & 0.020 & -0.013 & 0.023 & 0.084 & 0.070 & 0.054 & -0.016 & 0.027 & -0.164 & 0.157 & 0.020 & -0.014 & -0.044 & 0.000 & 0.147 \\
\hline $\begin{array}{l}\text { No. of } \\
\text { seeds/fruit }\end{array}$ & -0.022 & 0.026 & 0.003 & -0.043 & 0.266 & 0.063 & 0.025 & -0.084 & -0.004 & 0.043 & -0.008 & 0.014 & -0.042 & 0.618 & -0.028 & -0.011 & -0.008 & 0.020 & 0.846 \\
\hline $\begin{array}{l}\text { TSS ( } \\
\text { Brix) } \\
\end{array}$ & -0.051 & 0.120 & 0.002 & -0.061 & 0.426 & -0.096 & 0.039 & -0.046 & -0.006 & -0.035 & 0.032 & -0.047 & 0.023 & 0.119 & -0.144 & 0.074 & 0.057 & 0.023 & 0.419 \\
\hline $\begin{array}{l}\text { Ascorbic } \\
\text { acid } \\
\text { (mg/100g } \\
\text { Fwt.) } \\
\end{array}$ & -0.074 & -0.018 & 0.000 & 0.008 & -0.003 & 0.066 & -0.020 & 0.096 & 0.126 & 0.058 & -0.011 & 0.027 & -0.020 & 0.060 & 0.091 & -0.116 & -0.041 & -0.159 & 0.065 \\
\hline $\begin{array}{l}\text { Acidity } \\
(\%)\end{array}$ & 0.011 & -0.011 & 0.000 & -0.017 & -0.133 & 0.107 & -0.009 & 0.022 & 0.011 & 0.015 & 0.001 & 0.029 & -0.080 & 0.053 & 0.091 & -0.052 & -0.091 & -0.001 & -0.076 \\
\hline $\begin{array}{l}\text { Lycopene } \\
\text { (mg/100g } \\
\text { Fwt.) }\end{array}$ & -0.039 & -0.027 & 0.000 & 0.018 & 0.091 & 0.116 & -0.041 & 0.057 & 0.058 & 0.075 & 0.037 & -0.004 & 0.000 & 0.048 & 0.013 & -0.070 & 0.000 & -0.264 & -0.031 \\
\hline
\end{tabular}

Residual effect: 0.1945

* Significant at $1 \%, * *$ Significant at $5 \%$ 
Table.4 Direct (diagonal) and indirect effects of different qualitative and quantitative characters on yield in tomato at phenotypic level

\begin{tabular}{|c|c|c|c|c|c|c|c|c|c|c|c|c|c|c|c|c|c|c|c|}
\hline Parameters & $\begin{array}{c}\text { Days to } \\
1^{\text {st }} \\
\text { flowering }\end{array}$ & $\begin{array}{l}\text { No. of } \\
\text { flowers/ } \\
\text { cluster }\end{array}$ & $\begin{array}{c}\text { Days } \\
\text { to1 }^{\text {st }} \\
\text { harvest }^{-1}\end{array}$ & $\begin{array}{l}\text { No. of } \\
\text { clusters/ } \\
\text { plant }\end{array}$ & $\begin{array}{l}\text { No. of } \\
\text { fruits/ } \\
\text { cluster }\end{array}$ & $\begin{array}{l}\text { Fruit } \\
\text { dia. } \\
(\mathrm{cm})\end{array}$ & $\begin{array}{l}\text { Fruit } \\
\text { length } \\
\text { (cm) }\end{array}$ & $\begin{array}{c}\text { Avg } \\
\text { fruit } \\
\text { wt. } \\
\text { (g.) }\end{array}$ & $\begin{array}{l}\text { No. of } \\
\text { fruits/ } \\
\text { plant }\end{array}$ & $\begin{array}{l}\text { No. of } \\
\text { locules/ } \\
\text { fruit }\end{array}$ & $\begin{array}{l}\text { Plant } \\
\text { ht. } \\
(\mathrm{cm})\end{array}$ & $\begin{array}{c}\text { Pericarp } \\
\text { thickness } \\
(\mathrm{cm})\end{array}$ & $\begin{array}{c}\text { No. of } \\
\text { branches/ } \\
\text { plant }\end{array}$ & $\begin{array}{l}\text { No. of } \\
\text { seeds/ } \\
\text { fruit }\end{array}$ & $\begin{array}{c}\text { TSS } \\
\text { Brix })\end{array}$ & $\begin{array}{c}\text { Ascorbic } \\
\text { acid } \\
\text { (mg/100g } \\
\text { Fwt.) }\end{array}$ & $\begin{array}{l}\text { Acidity } \\
(\%)\end{array}$ & $\begin{array}{c}\text { Lycopene } \\
\text { (mg/100g } \\
\text { Fwt.) }\end{array}$ & $\begin{array}{c}\text { R value } \\
\text { (phenotypic } \\
\text { correlation) } \\
\text { with yield }\end{array}$ \\
\hline $\begin{array}{l}\text { Days to } 1^{\text {st }} \\
\text { flowering }\end{array}$ & -0.073 & -0.006 & -0.023 & -0.005 & -0.001 & -0.001 & -0.001 & 0.005 & -0.067 & -0.009 & 0.013 & 0.000 & 0.004 & $\begin{array}{c}- \\
0.069\end{array}$ & $\begin{array}{c}- \\
0.001\end{array}$ & 0.011 & -0.007 & 0.018 & -0.212 \\
\hline $\begin{array}{l}\text { No. of } \\
\text { flowers/ } \\
\text { cluster }\end{array}$ & 0.006 & 0.075 & 0.010 & 0.010 & 0.021 & 0.000 & -0.005 & $-\overline{-}$ & 0.025 & 0.001 & 0.008 & 0.013 & -0.008 & 0.113 & 0.002 & 0.006 & 0.003 & 0.002 & 0.267 \\
\hline $\begin{array}{l}\text { Days to1 }{ }^{\text {st }} \\
\text { harvest }\end{array}$ & -0.016 & -0.007 & -0.104 & -0.010 & -0.011 & -0.001 & 0.013 & 0.015 & -0.041 & -0.011 & $\begin{array}{c}- \\
0.032\end{array}$ & -0.011 & 0.019 & - & $\begin{array}{c}- \\
0.003\end{array}$ & -0.006 & -0.004 & 0.002 & -0.364 \\
\hline $\begin{array}{l}\begin{array}{l}\text { No. of } \\
\text { clusters/ } \\
\text { plant }\end{array} \\
\end{array}$ & 0.011 & 0.023 & 0.031 & 0.032 & 0.009 & 0.001 & -0.009 & $\overline{-}-\overline{018}$ & 0.053 & 0.006 & $-\overline{-}$ & 0.018 & -0.024 & 0.145 & 0.002 & 0.004 & 0.001 & 0.004 & 0.286 \\
\hline $\begin{array}{l}\text { No. of } \\
\text { fruits/ } \\
\text { cluster }\end{array}$ & 0.001 & 0.024 & 0.017 & 0.004 & 0.066 & 0.000 & -0.012 & 0.021 & 0.022 & 0.003 & 0.018 & -0.004 & -0.006 & 0.278 & 0.004 & -0.002 & 0.016 & -0.006 & 0.444 \\
\hline $\begin{array}{l}\text { Fruit dia. } \\
(\mathrm{cm})\end{array}$ & 0.013 & -0.005 & 0.020 & 0.003 & -0.001 & 0.007 & -0.004 & 0.010 & 0.025 & 0.012 & 0.025 & 0.012 & -0.005 & 0.134 & $\begin{array}{c}- \\
0.002\end{array}$ & -0.018 & -0.019 & -0.025 & 0.180 \\
\hline $\begin{array}{l}\text { Fruit } \\
\text { length(cm) }\end{array}$ & -0.001 & 0.008 & 0.028 & 0.006 & 0.016 & 0.001 & -0.049 & 0.026 & -0.005 & 0.001 & 0.013 & 0.007 & -0.017 & 0.158 & 0.005 & 0.012 & 0.005 & 0.027 & 0.245 \\
\hline $\begin{array}{l}\text { Avg fruit } \\
\text { wt. (g.) }\end{array}$ & -0.004 & -0.006 & -0.015 & -0.006 & 0.013 & 0.001 & -0.012 & 0.103 & -0.087 & -0.010 & $\begin{array}{c}- \\
0.003\end{array}$ & -0.033 & 0.021 & 0.215 & 0.001 & 0.017 & 0.006 & 0.023 & 0.235 \\
\hline $\begin{array}{l}\text { No. of } \\
\text { fruits/plant }\end{array}$ & 0.026 & 0.010 & 0.022 & 0.009 & 0.008 & 0.001 & 0.001 & $\begin{array}{c}- \\
0.047\end{array}$ & 0.189 & 0.015 & $\begin{array}{c}- \\
0.019\end{array}$ & 0.035 & -0.021 & $-\overline{-}$ & 0.000 & -0.026 & -0.002 & -0.022 & 0.162 \\
\hline $\begin{array}{l}\text { No. of } \\
\text { locules/ } \\
\text { fruit }\end{array}$ & 0.020 & 0.003 & 0.037 & 0.007 & 0.006 & 0.003 & -0.001 & $\begin{array}{c}- \\
0.031\end{array}$ & 0.090 & 0.032 & 0.011 & 0.031 & -0.028 & 0.153 & 0.000 & -0.020 & 0.000 & -0.013 & 0.303 \\
\hline $\begin{array}{l}\text { Plant ht. } \\
\text { (cm) } \\
\end{array}$ & -0.005 & 0.003 & 0.019 & -0.001 & 0.007 & 0.001 & -0.004 & $\stackrel{-}{0.002}$ & -0.021 & 0.002 & 0.177 & -0.011 & 0.005 & $-\overline{-}$ & 0.003 & 0.003 & -0.004 & -0.023 & 0.100 \\
\hline $\begin{array}{l}\text { Pericarp } \\
\text { thickness } \\
\text { (cm) }\end{array}$ & 0.000 & -0.010 & -0.012 & -0.006 & 0.002 & -0.001 & 0.003 & 0.033 & -0.065 & -0.010 & 0.020 & -0.102 & 0.025 & $\begin{array}{c}- \\
0.094\end{array}$ & 0.003 & 0.018 & 0.017 & 0.007 & -0.169 \\
\hline $\begin{array}{l}\text { No. of } \\
\text { branches/ } \\
\text { plant }\end{array}$ & 0.003 & 0.006 & 0.022 & 0.009 & 0.005 & 0.000 & -0.009 & 0.024 & 0.044 & 0.010 & 0.009 & 0.028 & -0.091 & 0.141 & 0.001 & -0.011 & -0.020 & 0.020 & 0.119 \\
\hline $\begin{array}{l}\text { No. of } \\
\text { seeds/fruit }\end{array}$ & 0.008 & 0.013 & 0.024 & 0.007 & 0.028 & 0.001 & -0.012 & 0.033 & -0.003 & 0.007 & $\overline{-}$ & 0.014 & -0.019 & 0.669 & 0.001 & -0.005 & -0.007 & 0.004 & 0.766 \\
\hline TSS (" Brix) & 0.006 & 0.008 & 0.024 & 0.005 & 0.017 & -0.001 & -0.016 & 0.007 & -0.003 & 0.000 & 0.035 & -0.024 & -0.003 & 0.054 & 0.014 & 0.015 & 0.019 & -0.004 & 0.153 \\
\hline $\begin{array}{l}\text { Ascorbic } \\
\text { acid } \\
\text { (mg/100g } \\
\text { Fwt.) } \\
\end{array}$ & 0.013 & -0.007 & -0.010 & -0.002 & 0.003 & 0.002 & 0.010 & $\begin{array}{c}- \\
0.029\end{array}$ & 0.080 & 0.010 & $0 . \overline{-}$ & 0.030 & -0.015 & 0.055 & 0.003 & -0.062 & -0.022 & -0.029 & 0.011 \\
\hline $\begin{array}{l}\text { Acidity } \\
(\%)\end{array}$ & -0.007 & -0.003 & -0.005 & 0.000 & -0.013 & 0.002 & 0.003 & $\begin{array}{c}- \\
0.008\end{array}$ & 0.005 & 0.000 & 0.009 & 0.023 & -0.024 & 0.058 & $\overline{-} \cdot \overline{0}$ & -0.018 & -0.077 & -0.009 & -0.080 \\
\hline $\begin{array}{l}\text { Lycopene } \\
\text { (mg/100g } \\
\text { Fwt.) }\end{array}$ & 0.009 & -0.001 & 0.002 & -0.001 & 0.003 & 0.001 & 0.009 & 0.017 & 0.029 & 0.003 & 0.029 & 0.005 & 0.013 & $\overline{0.020}$ & 0.000 & -0.013 & -0.005 & -0.142 & -0.097 \\
\hline
\end{tabular}

Residual effect: 0.2662

* Significant at $1 \%, * *$ Significant at $5 \%$ 
Number of clusters per plant had positive association with number of fruits per plant and TSS and negative association with days to first fruit harvest and shelf life.

\section{Path coefficient analysis}

Path coefficient analysis techniques devised by Dewey and Lu (1959) used to partition the correlation coefficient into direct and indirect effects of different characters on yield. Such information provides a realistic basis for allocation of appropriate weightage of various attributes while designing a pragmatic breeding programme for improvement of yield. The phenotypic and genotypic correlation coefficients between yield and other traits have been partitioned into direct and indirect effects by path coefficient analysis (Kumar and Singh, 2017d). In genotypic path coefficient (Table 3), maximum exerted positive and negative direct effects on fruit yield per plant was number of seeds per fruit (0.618) and lycopene content ($0.264)$, respectively. Whereas, high positive and negative indirect effects were expressed in TSS (0.426) via. number of fruits per cluster and days to $1^{\text {st }}$ harvest $(-0.240)$ via. number seeds per fruit, respectively. The estimate of residual effect $(0.1945)$ was low.

However, phenotypic path coefficient (Table 4), maximum exerted positive direct effects on fruit yield per plant was number of seeds per fruit (0.669) and maximum negative direct effects on fruit yield per plant was lycopene content (-0.142), whereas higher positive indirect effects were associated with number of fruits per cluster (0.278), average fruit weight (0.215), fruit length (0.158), number of locules per fruit (0.153) and number of branches per plant (0.141) via. number of seeds per fruit. However, higher negative indirect effects were expressed in days to first harvesting $(-0.154)$ via. number of seeds per fruit. The estimate of residual effect $(0.2662)$ was low. Estimation of correlations does not alone provide comprehensive pictures of the direct and indirect influence of each character to the yield. Path coefficient analysis is a tool for partition the observed correlation coefficient into direct as well as indirect effects of yield components or fruit yield per plant to provide clear picture of character association for formulating efficient selection strategy. Path analysis differs from simple correlation in that it points out the causes and their relative importance. The above mentioned results are in conformity to findings of Monamodi, et al., (2013), Alirwar et al., (2013), Reddy et al., (2013) and Laxmi et al., (2017).

As yield is a complex polygenic character influenced by contributions from different component traits, which are under separate genetic control, thus, it is essential to have a clear picture of the contributions of each of the component characters towards the ultimate yield. The correlation between yield and its components is indispensable when it is required to amalgamate high yield potential with other desirable traits in a single genotype.

\section{References}

Alirwar CS, Bahadur V, Prakash V. 2013. Genetic variability, heritability and correlation studies in tomato genotypes (Lycopersicon esculentum Mill.). Int. J. Agric. Sci. 9: 172-176.

Al-Jibouri, H. A., Miller, P.A. and Robinson, H. F. 1958. Genotypic and environmental variances and covariances in an upland cotton cross of interspecific origin. Agron. J. 50: 633636.

Deway, D. R. and Lu, K. N. 1959. A correlation and path coefficient analysis of components of crested wheat grass seed production. Agron. J. 51: 515-518. 
Kumar, C. and Singh, S.P. 2016a. Analysis of Combining Ability to recognize superior $F_{1}$ Hybrids in Tomato (Lycopersicum esculentum L.) for Yield and its contributing traits. The Bioscan 11(2): 949-952.

Kumar, C. and Singh, S.P. 2016b. Heterosis and inbreeding depression to identify superior $\mathrm{F}_{1}$ hybrids in tomato (Solanum lycopersicum L.) for the yield and its contributing traits. J. of Applied and Natural Sci. 8(1): 290-296.

Kumar, C. and Singh, S.P. 2017c. Heterosis and resistance against tomato leaf curl virus disease in tomato (Solanum lycopersicum L.). Agric Res J. 54(4): $469-474$.

Kumar, C. and Singh, S.P. 2017d. Estimation of combining ability analysis in tomato (Solanum lycopersicum L.) for yield, nutritional and processing quality improvement. J. of Applied and Natural Sci. 9 (4): 2021 -2025.

Lakshmi, E., Gasti, D.V. and Mulge, R. 2017. Character Interrelationship of Yield and
Yield Components in $\mathrm{F}_{2}$ Generation of Tomato (Solanum lycopersicum L.). Int.J.Curr.Microbiol.App.Sci. 6(11): 2351-2359.

Monamodi, E. L., Lungu, D. M. and Fite, G. L. 2013. Analysis of fruit yield and its components in determinate tomato (Lycopersicon lycopersci) using correlation and path coefficient, Botswana J. Agric. Appl. Sci. 9(1): 2940.

Ranganna, S. 1986. Handbook of Analysis and quality Control for Fruit and Vegtable products, Tata McGraw-Hill Education. Pp 92-95.

Reddy, R., Mula, B., Pratapa, R., Siddeswar, R., Hameedunnisa, B. 2013. Correlation and path analysis studies for yield and quality traits in tomato (Solanum lycopersicum L.), J. Agri. Veter. Sci. 4: 56-59.

Wright, S. 1921. Correlation and causation. $J$. Agricul. Res. 20: 557-587.

\section{How to cite this article:}

Chandan Kumar and Dheeraj Singh. 2018. A Correlation and Path Analysis Studies of Quantitative and Qualitative Traits in Tomato. Int.J.Curr.Microbiol.App.Sci. 7(04): 2230-2238. doi: https://doi.org/10.20546/ijcmas.2018.704.254 